
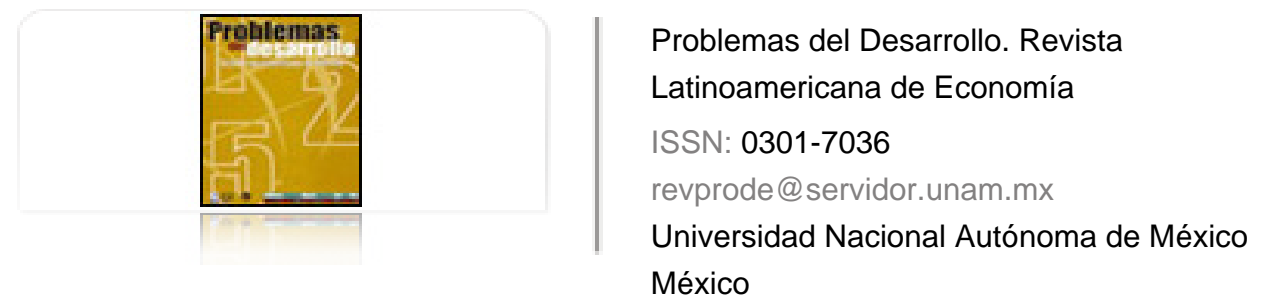

Sheinbaum Pardo, Claudia; Rodríguez Padilla, Víctor; Robles Morales, Guillermo POLÍTICA MEXICANA E INDICADORES DE SUSTENTABILIDAD

Problemas del Desarrollo. Revista Latinoamericana de Economía, vol. 40, núm. 158, julio-septiembre, 2009, pp. 113-135

Universidad Nacional Autónoma de México

Distrito Federal, México

Disponible en: http://www.redalyc.org/articulo.oa?id=11820200005

Cómo citar el artículo

- Número completo

- Más información del artículo

Página de la revista en redalyc.org

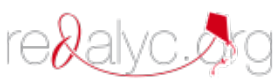

Sistema de Información Científica

Red de Revistas Científicas de América Latina, el Caribe, España y Portugal Proyecto académico sin fines de lucro, desarrollado bajo la iniciativa de acceso abierto 


\title{
POLITICA MEXICANA E INDICADORES DE SUSTENTABILIDAD
}

\author{
Claudia Sheinbaum Pardo* \\ Víctor Rodríguez Padilla** \\ Guillermo Robles Morales***
}

Fecha de recepción: 1 de octubre de 2008. Fecha de aceptación: 3 de marzo de 2009.

\section{Resumen}

El presente artículo analiza la política energética nacional y sus implicaciones en el marco del desarrollo sustentable, a través de una adaptación de la metodología propuesta por la Comisión Económica para América Latina y el Caribe junto con otras organizaciones, para los temas de autarquía, robustez, productividad, cobertura eléctrica, satisfacción de necesidades energéticas básicas, pureza relativa en el uso de la energía, participación de las fuentes renovables y alcance de recursos fósiles. El resultado muestra que a pesar de que algunos indicadores muestran mejoría, el valor promedio resulta menor para 2006 que para 1997, lo que indica un alejamiento del sistema energético mexicano de los criterios de sustentabilidad.

Palabras clave: energía, desarrollo sustentable, indicadores, México, política energética.

\begin{abstract}
This article analyzes Mexico's national energy policy, especially its implications within the framework of sustainable development, using a methodology adapted on the proposal of the Economic Commission for Latin America and the Caribbean, together with other organizations, and designed around the issues of autarchy, robustness, productivity, electricity coverage, satisfaction of basic energy needs, relative purity in the use of energy, participation of renewable sources and the scope for fossil sources. The result is that although some indicators show an improvement, the average value for 2006 is less than in 1997, pointing to the distancing of Mexico's energy system from sustainability criteria.

Keywords: energy, sustainable development, indicators, Mexico, energy policy.

* Investigadora titular A del Instituto de Ingeniería de la UNAM.

Correo electrónico: csp@ pumas.iingen.unam.mx

** Investigador titular B del posgrado de la Facultad de Ingeniería de la UNAM.

Correo electrónico: energia123@hotmail.com

***Académico del Instituto de Ingeniería de la UNAM.

Correo electrónico: grom@pumas.iingen.unam.mx
\end{abstract}




\section{Résumé}

Le présent article analyse la politique énergétique nationale, plus particulièrement ses implications en ce qui concerne le développement durable, à travers une méthodologie adaptée de celle proposée par la Comission Économique pour l'Amérique Latine et les Caraïbes ainsi que d'autres organisations, et élaborée pour englober les thèmes d'autarcie, robustesse, productivité, couverture des besoins en électricité, satisfaction des besoins basiques en énergie, pureté relative dans l'usage de l'énergie, recours aux sources renouvelables et réserves de ressources fossiles. Le résultat indique que, bien qu'on puisse noter une amélioration de certains indicateurs, la valeur moyenne pour 2006 est inférieure à celle de 1997, ce qui montre que le système énergétique mexicain s'éloigne des critères de durabilité.

Mots clés: énergie, développement durable, indicateurs, Mexique, politique énergétique.

\section{Resumo}

Este artigo analisa a política energética nacional, especialmente suas implicações no marco do desenvolvimento sustentável, através de uma metodologia adaptada da proposta pela Comissão Econômica para a América Latina e o Caribe junto com outras organizações, e desenhada para os temas de autarquia, robustez, produtividade, cobertura elétrica, satisfação de necessidades energéticas básicas, pureza relativa no uso da energia, participação das fontes renováveis e alcance de recursos fósseis. $O$ resultado mostra que, apesar de que alguns indicadores mostram melhora, o valor médio para 2006 é menor que o de 1997, o que mostra o distanciamento do sistema energético mexicano dos critérios de sustentabilidade.

Palavras-chave: energia, desenvolvimento sustentável, indicadores, México, política energética. 


\section{Introducción}

oda política energética persigue objetivos generales y específicos: los primeros están asociados a las preocupaciones de desarrollo nacional y humano; los segundos, a los problemas que aparecen a lo largo de las cadenas de suministro. Para satisfacerlos, las autoridades tutelares del sector establecen grandes líneas de acción o estrategias, que movilizan un conjunto de instrumentos de jerarquía y naturaleza diversas, desde la intervención directa por parte del Estado, hasta mecanismos inductivos o de fomento para estimular a los agentes a desenvolverse de manera favorable a los objetivos de las políticas públicas en la materia. A partir de la década de 1990 y bajo el marco de la orientación del desarrollo sustentable, la política energética ha comenzado a evaluarse desde esta perspectiva.

El desarrollo sustentable fue definido por la Comisión Brundtland como aquel

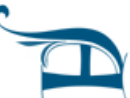
que satisface las necesidades del presente sin comprometer la habilidad de las futuras generaciones para satisfacer las propias (WCED, 1987). Acorde con este planteamiento, la Cumbre de la Tierra de 1992 estableció la necesidad de encontrar un punto de equilibrio entre las exigencias económicas, sociales y ambientales tanto de las generaciones presentes como futuras.

El tema energético ha sido parte fundamental en el debate sobre el desarrollo sustentable. La disponibilidad de recursos energéticos es indispensable para resolver la pobreza y mejorar las condiciones de vida, es clave en la economía de las naciones, pero la producción y el uso de los recursos energéticos constituyen actividades que generan la mayor parte de los impactos ambientales locales y globales.

En 1987, año en que se publicó el reporte de la Comisión Brundtland, Goldemberg, Johanson, Reddy y Williams (1987a: 5; 1987b: 6) publicaron dos libros, Energy for Development y Energy for a Sustainable World, donde plantearon la necesidad de crear esquemas de desarrollo que mejoraran las condiciones de vida de la población, pero que fueran menos intensivos en el empleo de la energía, tanto por razones ambientales como de desarrollo económico y social, particularmente en los países pobres.

Esta perspectiva apunta al reconocimiento de la energía no como un fin en sí mismo, sino como un medio para alcanzar mejores niveles de vida. Diversas agencias especializadas en energía, International Atomic Energy Agency (IAEA), United Nations Department of Economic and Social Affairs (UNDESA), International Energy Agency (IEA), Eurostat y European Energy Agency (EEA), recuperan esta idea en un documento publicado recientemente (IAEA, UNDESA, IEA, Eurostat, EEA, 2005: 1-2): "Aun cuando sea fundamental para el desarrollo, la energía es sólo un medio hacia un fin. El fin es buena salud, altos niveles de vida, una economía sustentable y un am- 
biente limpio. Ninguna forma de energía -carbón, solar, nuclear, viento o cualquier otra- es buena o mala en sí misma, y cada una debe ser evaluada en la medida que pueda proveer dichos fines".

Este enunciado se relaciona con uno de los temas de debate internacional sobre el desarrollo sustentable, a saber, la necesidad de establecer criterios, metas e indicadores que permitan medirlo. Estos esfuerzos se plantearon como una necesidad en la Agenda 21 adoptada en la Cumbre de la Tierra (CSD, 1992), en particular en el capítulo 40, que demanda a los países, organizaciones gubernamentales y no-gubernamentales a desarrollar el concepto de indicadores y su armonización en los ámbitos nacional, regional y global.

En 1995, el Departamento de Asuntos Económicos y Sociales de Naciones Unidas comenzó a trabajar en la creación de indicadores que consideran cuatro grandes dimensiones: social, económica, ambiental e institucional. El grupo de expertos en indicadores de desarrollo sustentable de este organismo ha venido perfeccionando su trabajo, de tal forma que en la última versión incluye 58 indicadores clasificados en cuatro dimensiones, 15 temas y 38 subtemas (UNDESA, 2001).

En este mismo sentido, ha habido esfuerzos por determinar indicadores para evaluar la sustentabilidad de los sistemas energéticos. Uno de ellos fue presentado por la Agencia Internacional de Energía Atómica en colaboración con otros organismos en la novena sesión de la Comisión para el Desarrollo Sustentable de Naciones Unidas, cuya actualización fue publicada en 2005 (IAEA, UNDESA, IEA, Eurostat, EEA, 2005). Otro ejemplo es el elaborado por la Comisión Económica para América Latina y el Caribe, la Organización Latinoamericana de Energía y la Sociedad Alemana de Cooperación Técnica (CEPAL, OLADE, GTZ, 2003: 47; descrito también en Salgado, Altomonte, 2001: 10-15 ).

El presente trabajo analiza la sustentabilidad del sistema energético mexicano basado en los indicadores planteados por CEPAL et al. (2003) para los años 1997 y 2006. Cabe mencionar que si bien los temas e indicadores que presenta la metodología de IAEA et al. (2005), tienen una mayor desagregación en términos de los sectores de consumo final de la energía, usos finales e impacto en atmósfera, agua y suelo (tabla A); los indicadores de CEPAL et al. (2003) reconocen con mayor amplitud las dimensiones económicas y políticas, de sumo interés para el análisis del sistema energético mexicano. La tabla B muestra los indicadores que considera la metodología de CEPAL et al. (2003). 
El artículo consta de cinco partes. Luego de esta introducción se ofrece un análisis general de lo que ha sido la política energética mexicana; más adelante se presenta la metodología y definición de indicadores de sustentabilidad, el análisis de la sustentabilidad del sistema energético mexicano, los resultados y conclusiones.

\section{La política energética mexicana de la última década}

Desde 1982, las diferentes administraciones federales han buscado solucionar los problemas y cumplir con los objetivos generales y específicos del sector energético mediante cinco grandes líneas de política energética, que no son precisamente las recomendadas por las agencias internacionales en el marco del desarrollo sustentable, sino las que responden a los objetivos de apertura del mercado y la integración de América del Norte.

1. Cambio de modalidad de coordinación sectorial. ${ }^{1}$ El modelo de control central -caracterizado por la exclusividad del Estado en las decisiones, formuladas sobre la base de una planificación centralizada de carácter normativo y la prioridad política sobre su ejecución por parte de empresas estatales- se reemplaza por un modelo de mercado abierto. Tal reemplazo presupone un proceso de reorganización industrial, que incluye la desintegración vertical y horizontal de las cadenas productivas; la liberalización de la inversión y el comercio (interno y externo); la enajenación de activos del Estado; la búsqueda de una estructura organizacional de los organismos públicos del sector (Pemex, CFE y LFC) similar a los corporativos transnacionales; y la creación de nuevas regulaciones e instituciones de mercado. ${ }^{2}$

1 El concepto de modalidades de coordinación sectorial se refiere esencialmente a tres aspectos estrechamente interrelacionados: las formas de organizar institucionalmente o de coordinar las decisiones de asignación de los recursos en el ámbito de cada cadena productiva y el sistema energético en su conjunto; el tipo de racionalidad que orienta a dichas decisiones, es decir, a las finalidades principales que se pretenden alcanzar; los esquemas utilizados para regular el funcionamiento de los mercados del sector. Véase: OLADE, CEPAL, GTZ, "Energía y desarrollo sustentable en América Latina y el Caribe: Guía para la formulación de políticas energéticas", OLADE, Quito, Ecuador, julio de 2000.

2 La liberalización del comercio y la inversión, que ha tocado casi todas las actividades productivas del país (telecomunicaciones, bancos, ferrocarriles, puertos, aeropuertos, agua potable, drenaje, ingenios, etc.), tiene su origen en una visión teórico-normativa que asegura que la competencia en el mercado tiene virtudes que no podría conseguir la injerencia directa del Estado (mediante empresas públicas) o una intervención indirecta mediante regulaciones restrictivas. Entre esas virtudes se encuentran la eficiencia productiva, la productividad, la disponibilidad de capital. Desde esa perspectiva, todas las actividades productivas, incluyendo las que requieren infraestructura pesada en situación de monopolio natural, son contestables y podrían realizarse mediante mecanismos de mercado. Los gobiernos que se han sucedido desde 1982, han adoptado ese enfoque, ya sea por convicción o por recomendación de los organismos financieros internacionales. 
2. Promoción del gas natural. El gas natural se ha promovido intensamente como sustituto de otras fuentes de energía en todos los sectores de consumo, especialmente en la generación de electricidad, en razón de su supuesto bajo costo, ${ }^{3}$ menor impacto ambiental, así como mayor versatilidad y elevada eficiencia en su uso.

3. Concentración de los recursos públicos en la extracción de petróleo crudo y empleo de capital privado en casi todas las demás actividades a cargo del Estado, aprovechando novedosos esquemas financieros que desde 1997 se han agrupado en la modalidad de Programas de Inversión con Registro Diferido en el Gasto o Pidiregas. La justificación principal de dicha política ha sido la supuesta búsqueda de rentabilidad de la inversión pública y el interés manifiesto del sector privado por las actividades del sector energético.

4. Utilización de Pemex y de la CFE como instrumentos al servicio de las políticas de estabilidad macroeconómica. Esto es posible en virtud de su estatus jurídico (bajo control total del Estado) y la magnitud de sus presupuestos de ingresos y de egresos (en ambos casos definidos por el Estado).

5. Búsqueda de la seguridad energética ya no en el ámbito nacional sino en un marco regional, que comprende el círculo de la seguridad energética de América del Norte, mediante la consolidación de los vínculos con los Estados Unidos y Canadá, a través de la Alianza para la Seguridad y Prosperidad de América del Norte (ASPAN).

En términos generales, estas cinco líneas de política energética han debilitado al sector, tanto a la luz de los indicadores de sustentabilidad energética, como en términos de una política energética de Estado que fortalezca la seguridad nacional.

El cambio en la modalidad de la coordinación sectorial se ha traducido en el debilitamiento de los organismos públicos, pues ha implicado ajustes en Pemex, CFE y LFC, para dar acomodo a la competencia y al desenvolvimiento del sector privado. Los ajustes han consistido en la segmentación organizativa, la cesión de partes de mercado (construcción de infraestructura, generación de electricidad, distribución de gas natural), el inmovilismo para dejar que el sector privado aproveche las nuevas oportunidades de negocio (suministro de gas natural licuado, telecomunicaciones utilizando la red de fibra óptica) ${ }^{4}$.

Lo cual dejó de cumplirse desde el año 2000.

4 La reciente reforma energética aprobada en octubre de 2008 apuesta a la contratación excesiva de servicios por parte de Pemex, y dado que la voluntad de la administración actual está en la apertura, el deterioro de las empresas energéticas nacionales será cada vez mayor. 
Asimismo, esta política se ha traducido en el deterioro de las finanzas del sector, provocado por la convergencia de varios factores: 1) disminución de los recursos presupuestales destinados a actividades diferentes a la extracción de petróleo y gas; 2) decisión de contratar la mayor parte de las obras y servicios mediante endeudamiento; 3) incorporación desproporcionada de los pasivos laborales y ambientales en la contabilidad de los organismos; $;{ }^{4}$ ) excesiva carga fiscal (que obliga a Pemex a endeudarse para pagar los impuestos, y en el caso de la CFE ha requerido cuantiosos subsidios que afectan negativamente su situación patrimonial); 5) controles desmesurados; y 6) condiciones costosas de intercambio entre los organismos ${ }^{6}$.

Cabe recordar que a finales de los noventa se buscó la liberalización total del sector eléctrico, cuando el ejecutivo federal intentó una reforma constitucional para crear un mercado eléctrico a partir del desmantelamiento y privatización de los operadores históricos del servicio público (CFE y LFC); sin embargo, dicha reforma provocó una fuerte resistencia entre diversos sectores del país, de manera que la orientación posterior fue hacia el incremento de la generación privada mediante productores independientes.

Esa voluntad de socavar las fortalezas de las empresas paraestatales también se ha traducido en el abandono paulatino de las actividades de investigación y desarrollo tecnológico. Simultáneamente, las limitaciones al crecimiento se han acompañado de medidas para operar un cambio cultural al interior de los organismos mediante la rápida renovación del personal (por medio de despidos y jubilaciones anticipadas). En Pemex, las consecuencias han sido graves, pues ha disminuido significativamente su capacidad de desarrollo de tecnología propia y de recursos humanos altamente calificados para abordar los nuevos retos como la explotación petrolera en aguas profundas.

En lo que se refiere a la promoción del gas natural, se pretende haber logrado cierto balance energético al reducir la dependencia del petróleo; no obstante, la sustitución se ha realizado con un solo combustible fósil y, además, sin contar con la producción nacional necesaria, lo que ha provocado que el peso de las importaciones en el consumo se haya incrementado. Además, hay que considerar que la penetración más rápida e importante del gas natural se ha dado en el sector eléctrico, con cen-

5 La contabilización de los pasivos laborales suponiendo una liquidación inmediata y completa de la plantilla de empleados y trabajadores ha sido cuestionada, entre otras razones, porque la ley no prevé la posibilidad de quiebra de los organismos y las promesas gubernamentales de excluir definitivamente la privatización.

$6 \quad$ LFC compra energía a la CFE a precios más altos de los que a ésta se le autoriza vender. La CFE compra combustibles a Pemex a precios más altos de los que pudiera conseguir en el mercado internacional. Pemex tiene un sistema de precios de transferencia entre organismos que hace incosteables las operaciones. 
trales de ciclo combinado que son propiedad del sector privado en la modalidad de productores independientes.

Por otro lado, se ha privilegiado la extracción de petróleo crudo en detrimento de las tareas de exploración, indispensables para renovar los volúmenes extraídos, lo que ha generado una importante caída de las reservas probadas. Al mismo tiempo, la tasa de recuperación del petróleo in situ ha sido baja, al no consentir inversiones sistemáticas en recuperación mejorada y asistida, fenómeno agravado por la obsolescencia del reglamento de trabajos petroleros.

La carencia de inversión en otras ramas de la industria petrolera como refinación y petroquímica, así como la escasez de materia prima al privilegiar la exportación de crudo, ha provocado la creciente importación de productos refinados y petroquímicos.

A lo anterior se aúna la deficiente infraestructura de transporte de combustibles líquidos, por falta de recursos en el mantenimiento, modernización y ampliación de la red de transporte y distribución por ductos, lo cual ha derivado en aumento de costos, accidentes fatales y daños al ambiente.

En lo referente al uso de los organismos como instrumentos al servicio de las políticas de estabilidad macroeconómica, se han generado cuantiosos ingresos para el Estado, pero pocos se han reinvertido en el sector energético. La mayor parte se ha destinado al gasto corriente, en particular se ha empleado para cubrir los hoyos de la evasión fiscal.

Finalmente, se ha priorizado una supuesta seguridad energética de América del Norte, a costa de la seguridad energética nacional, ampliando las exportaciones nacionales de crudo hacia los Estados Unidos e incrementando las importaciones de gas natural y derivados del petróleo. La integración ha promovido el establecimiento de los precios internos con base en referencias internacionales (Estados Unidos). Si bien ello ha permitido maximizar los ingresos fiscales, se han limitado las posibilidades de un desarrollo endógeno basado en un suministro de energía competitivo.

Adicionalmente, esta política ha nulificado el aprovechamiento de fuentes renovables de energía, a pesar de la contribución de México al cambio climático y de los problemas de contaminación local provocados por las fuentes fósiles. Por otra parte, la ampliación de la participación privada tanto en los sistemas de transporte y distribución de gas natural como en la generación eléctrica ha tenido como consecuencia mayores precios para los usuarios finales.

A los inconvenientes esbozados todavía hay que sumar la debilidad institucional. Por una parte, la Secretaría de Energía (Sener) no ha logrado consolidarse como la autoridad tutelar del sector, por su escaso peso político frente a una SHCP encargada de fijar los precios y tarifas de los combustibles, la electricidad y las materias primas 
petroquímicas, determinar los criterios de planeación del sector y autorizar los proyectos de inversión de los organismos públicos, así como sus presupuestos de ingresos y egresos. La Sener tampoco cuenta con poder real para obligar a los organismos públicos a coordinarse y unir esfuerzos alrededor del óptimo nacional: sus directores prefieren acordar directamente con el presidente y el secretario de Hacienda y Crédito Público. Un agravante adicional es la escasa solvencia técnica de la Sener, explicable por los continuos cambios de los equipos directivos y técnicos, que la hacen dependiente de la información y análisis proporcionados por los organismos públicos.

La reciente reforma energética aprobada en octubre de 2008 por el Congreso de la Unión poco abona al fortalecimiento del sector, debido a que la orientación de la administración actual está sustentada en los preceptos señalados previamente.

Estas políticas y sus consecuencias se analizan a continuación, en el marco de diversos indicadores de desarrollo sustentable para el sector energético.

\section{Evaluación de los indicadores de sustentabilidad energética para México}

Con base en la metodología de CEPAL et al. (2003: 47) y de acuerdo con la información disponible para evaluar el sistema energético mexicano, en este trabajo se utilizan los temas e indicadores presentados en la tabla 1. La evaluación y análisis se realiza para la década 1997-2006; no obstante, se han indicado los casos en que la falta de información nos ha obligado a cambiar los años estadiados.

tabla 1.

Indicadores de evaluación de sustentabilidad del sistema energético mexicano

\begin{tabular}{|l|l|}
\hline \multicolumn{2}{|c|}{ Temas } \\
\hline Autarquía energética & $\begin{array}{l}\text { Importación de energía primaria } \\
\text { Importación de hidrocarburos }\end{array}$ \\
\hline Robustez frente a cambios externos & $\begin{array}{l}\text { Exportaciones energéticas al PIB } \\
\text { Participación de las exportaciones de petróleo en los ingresos nacionales } \\
\text { Deuda del sector energético }\end{array}$ \\
\hline Productividad energética & Inverso de la intensidad energética (consumo nacional de energía /PIB) \\
\hline Cobertura eléctrica & Porcentaje de hogares con electricidad \\
\hline Cobertura de necesidades energéticas básicas & $\begin{array}{l}\text { Energía útil en el sector residencial } \\
\text { Gasto de energía por deciles }\end{array}$ \\
\hline Pureza relativa del uso de la energía & Emisiones de CO \\
\hline Uso de energías renovables & Participación de energías renovables \\
\hline Alcance recursos fósiles & Reservas de hidrocarburos entre producción \\
\hline
\end{tabular}

Fuente: CEPAL, OLADE, GTZ (1997). 
En el siguiente apartado se detalla cada uno de los indicadores y sus tendencias. Con la finalidad de apreciar mejor el resultado, así como de respetar la metodología de CEPAL et al. (2003: 50), cada indicador es normalizado en una escala de cero a 10, donde cero implica menor sustentabilidad. Los resultados se muestran en forma de radogramas, cuyos vértices representan a cada indicador.

\section{Evaluación}

La autarquía o autosuficiencia energética implica que el país pueda garantizar el suministro energético de largo plazo mediante recursos propios y, por lo tanto, cada vez dependa menos de las importaciones. En México, entre 1997 y 2006, la mayor parte del consumo de combustibles estuvo sustentada en las importaciones (tabla 2). En el caso de los derivados del petróleo, la causa fue la falta de inversión en nuevas refinerías y el crecimiento acelerado del consumo de gasolinas. En cuanto al gas natural, como ya se ha dicho, el incremento de las importaciones se debió a la decisión de sustentar la generación de electricidad en ese combustible, sin contar con las reservas ni la producción suficiente para soportar el crecimiento con producto nacional. Es de observarse que el peso de las importaciones ha aumentado precisamente en los combustibles de mayor demanda (gas natural, gasolinas y diesel). Asimismo, se han elevado las importaciones de carbón para la generación eléctrica, a pesar de que existen otras alternativas para el país, como el combustóleo, que ha disminuido su participación en la generación eléctrica, no obstante que los impactos ambientales de la quema de carbón son más perniciosos que los del combustóleo.

$\mathrm{Al}$ aumentar las importaciones de energía, la autarquía energética del país ha disminuido.

Tabla 2.

Peso relativo de las importaciones netas en

el consumo nacional de combustibles

\begin{tabular}{|l|c|c|}
\hline \multicolumn{2}{|c|}{1997} & 2006 \\
\hline Carbón & $37.2 \%$ & $63.0 \%$ \\
Coque de carbón & $0.0 \%$ & $7.1 \%$ \\
Coque de petróleo & $0.0 \%$ & $69.3 \%$ \\
Gas licuado & $23.8 \%$ & $25.3 \%$ \\
Gasolinas & $11.8 \%$ & $27.2 \%$ \\
Diesel & $2.9 \%$ & $10.8 \%$ \\
Combustóleo & $16.0 \%$ & $-18.0 \%$ \\
Gas seco & $2.5 \%$ & $20.9 \%$ \\
\hline \multirow{2}{*}{ TOTAL } & $\mathbf{9 . 4 \%}$ & $\mathbf{1 6 . 7 \%}$ \\
\hline
\end{tabular}

Fuente: elaboración propia a partir de datos de Sener (2006). 
La robustez es una categoría que expresa la vulnerabilidad económica del país ante cambios externos relacionados con el sector energético, como el precio internacional del crudo. Para medirla, se diseñaron tres indicadores: valor de las exportaciones de crudo respecto del PIB, participación del ingreso petrolero en el total y peso relativo del endeudamiento en la inversión del sector petrolero. Entre 1997 y 2006, el valor de las exportaciones respecto del PIB creció de $11.9 \%$ a 23.4\%, sin embargo, en unidades energéticas, pasó de 0.15 a 0.12 barriles anuales exportados por dólar de PIB. Por otro lado, la participación del ingreso petrolero respecto al total del ingreso del sector público pasó de $37.6 \%$ a 38.8\%, y la inversión en Pemex a través de deuda (Pidiregas) creció de $14.3 \%$ a $86.6 \%$ (Tabla 3). Claramente, el precio del barril de petróleo tiene un peso significativo, lo cual indica que la economía mexicana es sumamente vulnerable a cambios externos, particularmente al precio internacional del barril de petróleo.

Tabla 3.

Indicadores de robustez

\begin{tabular}{|c|c|c|c|c|c|c|c|c|c|c|c|}
\hline & & 1997 & 1998 & 1999 & 2000 & 2001 & 2002 & 2003 & 2004 & 2005 & 2006 \\
\hline$P I B$ & \begin{tabular}{|l|} 
millones de \\
pesos de 1993
\end{tabular} & 1387.5 & 1449.3 & 1505.4 & 1604.8 & 1602.3 & 1615.6 & 1637.4 & 1705.8 & 1753.8 & 1837.3 \\
\hline $\begin{array}{c}\text { Exportaciones } \\
\text { de crudo }\end{array}$ & \begin{tabular}{|l|} 
millones de \\
pesos de 1993
\end{tabular} & 163.8 & 102.0 & 136.6 & 207.2 & 165.0 & 178.5 & 238.2 & 300.4 & 379.3 & 430.4 \\
\hline Porcentaje & & $11.9 \%$ & $7.0 \%$ & $9.1 \%$ & $12.9 \%$ & $10.3 \%$ & $11.0 \%$ & $14.5 \%$ & $17.6 \%$ & $21.6 \%$ & $23.4 \%$ \\
\hline $\begin{array}{c}\text { Exportaciones } \\
\text { de crudo }\end{array}$ & $\begin{array}{l}\text { millones de } \\
\text { barriles diarios }\end{array}$ & 1.731 & 1.738 & 1.551 & 1.620 & 1.757 & 1.716 & 1.848 & 1.874 & 1.833 & 1.789 \\
\hline$E x p / P I B$ & $\begin{array}{l}\text { miles bb } \\
\text { anvales/US }\end{array}$ & 0.15 & 0.15 & 0.13 & 0.12 & 0.13 & 0.13 & 0.14 & 0.13 & 0.13 & 0.12 \\
\hline Ingreso total & $\begin{array}{l}\text { millones de } \\
\text { pesos corrientes }\end{array}$ & 508.7 & 545.2 & 674.3 & 868.3 & 939.1 & 989.4 & 1133.0 & 1270.2 & 1412.5 & 1558.8 \\
\hline $\begin{array}{l}\text { Ingreso } \\
\text { petrolero }\end{array}$ & $\begin{array}{l}\text { millones de } \\
\text { pesos corrientes }\end{array}$ & 181.5 & 170.9 & 209.9 & 319.4 & 318.7 & 293.0 & 397.6 & 491.1 & 592.3 & 605.4 \\
\hline Porcentaje & & $35.7 \%$ & $31.4 \%$ & $31.1 \%$ & $36.8 \%$ & $33.9 \%$ & $29.6 \%$ & $35.1 \%$ & $38.7 \%$ & $41.9 \%$ & $38.8 \%$ \\
\hline Producción & $\begin{array}{l}\text { millones de } \\
\text { barriles diarios }\end{array}$ & 3.025 & 3.073 & 2.909 & 3.015 & 3.130 & 3.180 & 3.375 & 3.385 & 3.335 & 3.258 \\
\hline Prod/Ingreso & $\begin{array}{l}\text { miles bb } \\
\text { anuales/US }\end{array}$ & 0.72 & 0.69 & 0.52 & 0.42 & 0.47 & 0.39 & 0.36 & 0.32 & 0.29 & 0.25 \\
\hline $\begin{array}{r}\text { Inversión no } \\
\text { Pidiregas }\end{array}$ & $\begin{array}{l}\text { millones de } \\
\text { pesos corrientes }\end{array}$ & 47.9 & 43.1 & 37.5 & 34.1 & 31.1 & 24.5 & 19.0 & 11.3 & 19.2 & 16.8 \\
\hline $\begin{array}{l}\text { Inversión } \\
\text { Pidiregas }\end{array}$ & $\begin{array}{l}\text { millones de } \\
\text { pesos corrientes }\end{array}$ & 8.0 & 27.5 & 34.3 & 50.1 & 42.7 & 61.7 & 94.7 & 102.4 & 94.5 & 108.7 \\
\hline $\begin{array}{l}\text { Pidiregas/ } \\
\text { inversión total }\end{array}$ & & $14.3 \%$ & $39.0 \%$ & $52.1 \%$ & $59.5 \%$ & $57.9 \%$ & $71.6 \%$ & $83.3 \%$ & $90.0 \%$ & $83.1 \%$ & $86.6 \%$ \\
\hline
\end{tabular}

Fuente: Banco de Información Económica, INEGI; Informe de Gobierno, 2007; Anuario estadístico Pemex, 2001 a 2007. 
El tercer indicador, la deuda, se ha incrementado significativamente en todo el periodo analizado.

Es claro que para todos los casos, los indicadores tuvieron una tendencia de crecimiento durante toda la década, aun cuando sus valores fluctuaron para algunos años. De tal forma que los resultados no pueden asociarse a las características específicas de determinado año, sino a un comportamiento tendencial del sector energético nacional durante la última década.

Productividad energética. La productividad se define como lo inverso a la intensidad energética, que a su vez puede medirse como el consumo nacional de energía ${ }^{7}$ por unidad de PIB. Mientras menor sea la intensidad energética, la productividad y eficiencia energética del país aumentan. En las últimas décadas, la intensidad energética nacional ha tenido un comportamiento errático (figura 1), sin embargo, en su valor total de 1997 a 2006, la intensidad disminuyó $2.6 \%$ y, por tanto, aumentó la productividad energética del país.

figura 1.

Intensidad energética

Consumo nacional de energía entre PIB

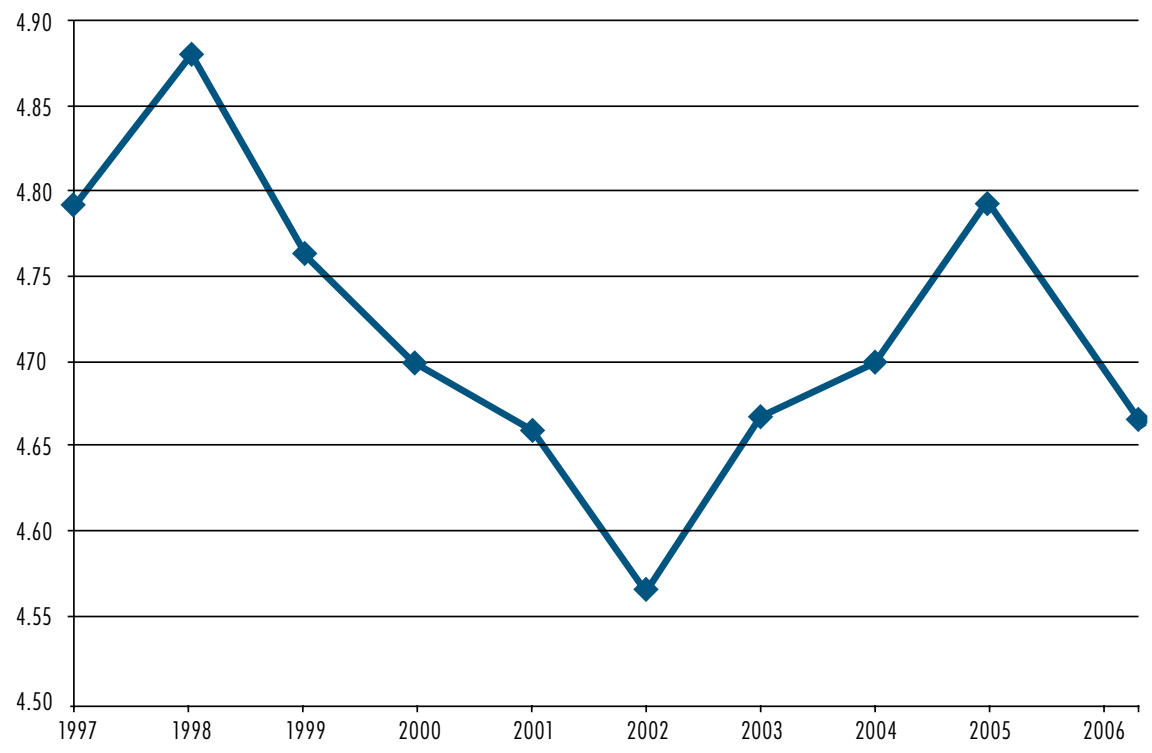

Fuente: Banco de Información Económica, Inegi.

7 El consumo nacional está compuesto por la suma del consumo de energía para el sector energético (consumo propio, consumo para transformación y pérdidas por distribución), las recirculaciones, las diferencias estadísticas y el consumo final total (consumo no energético y consumo energético).

\section{Desarrollo}


Cobertura eléctrica. De acuerdo con el informe de gobierno de 2007, entre 1995 y 2005 el porcentaje de viviendas con electricidad se elevó de $93 \%$ a 97\%. No obstante este crecimiento, hay aún 741000 viviendas -que equivalen a poco más de 3 millones de habitantes- que carecen de este servicio.

Cobertura de necesidades energéticas básicas. Para evaluar esta categoría se definen dos indicadores: eficiencia de transformación entre energía final y útil per cápita, y equidad en el gasto de energía por deciles, para diversas fuentes. La energía útil se define como la cantidad de energía que llega a la vivienda (energía final), multiplicada por la eficiencia en su uso. De acuerdo con la metodología de CEPAL (Salgado, Altomonte, 2001: 11), la energía útil se calcula conforme a las eficiencias establecidas por el Sistema de Información Económica y Energética (SIEE) de la Organización Latinoamericana de Energía (OLADE). Los valores asignados a las eficiencias son: electricidad (0.8), gas natural (0.5), GLP (0.4), querosina (0.35), leña (0.1).

La tabla 4 muestra el consumo per cápita de energía final y útil en el sector residencial para los años de estudio. Como se observa, la energía útil per cápita permanece constante pero la energía final per cápita disminuye, esto implica que la eficiencia (energía final/ energía útil per cápita) de la transformación aumentó de $36 \%$ a $39 \%$. Este cambio se debe a la modificación en la estructura de consumo por energía final (mix energético). Al disminuir la participación del GLP y aumentar la de la electricidad, el consumo es más eficiente en términos de su transformación de energía final a energía útil.

Tabla 6.

Energía útil per cápita (PJ/millones de habitantes)

\begin{tabular}{|l|r|r|r|r|}
\hline \multicolumn{5}{|c|}{1997} \\
\hline \\
Leña & Energía final & Energía útil & Energía final & Energía útil \\
GLP & 247.0 & 24.7 & 247.2 & 24.7 \\
Querosina & 307.4 & 122.9 & 266.5 & 106.6 \\
Gas natural & 2.1 & 0.7 & 1.8 & 0.6 \\
Electricidad & 22.4 & 11.2 & 29.6 & 14.8 \\
Total & 106.7 & 85.4 & 160.0 & 128.0 \\
Población (millones) & 685.5 & 244.9 & 705.2 & 274.8 \\
Per cápita & 93.6 & 93.6 & 104.0 & 104.0 \\
\hline
\end{tabular}

Fuente: Balance Nacional de Energía, 2007.

Eficiencias del BIEE-OLADE: electricidad (0.8), gas natural (0.5), GLP (0.4), querosina (0.35), leña (0.1); Salgado, Altomonte (2001:11). 
La equidad en el gasto de energía se presenta a través del índice de Gini, que muestra la concentración en el gasto de diferentes energéticos por decil de ingreso corriente monetario para los años 2000 y 2006. Si hay equidad en el gasto, el índice de Gini es igual a cero. La concentración máxima se presenta cuando éste es igual a uno.

Al evaluar el índice de Gini de acuerdo con la información de la Encuesta Ingreso Gasto de los Hogares, publicada por el INEGI (2008) para 2000 y 2006 (tabla 5), puede observarse que éste es menor para el gasto en electricidad, lo que representa una ligera mejoría en la equidad, y se mantiene casi constante para gas y otros combustibles, lo que indica que la inequidad es similar para ambos años.

tabla 5.

Índice de Gini del gasto por deciles de nivel de ingreso corriente monetario

\begin{tabular}{|l|l|l|}
\hline \multicolumn{2}{|c|}{2000} & 2006 \\
\hline Gasto en electricidad & 0.67 & 0.59 \\
Gasto en gas & 0.57 & 0.57 \\
Gasto en otros combustibles & 0.87 & 0.86 \\
\hline
\end{tabular}

Elaboración propia de acuerdo con ENIGH, 2000 y 2006.

La pureza relativa del uso de la energía se mide a través de las emisiones de bióxido de carbono, el principal gas de efecto invernadero que provoca el cambio climático global. Estas emisiones se calculan de acuerdo con la metodología del Panel Intergubernamental de Cambio Climático (IPCC, 1996) para el consumo de energía de los sectores de uso final (con excepción de los usos no energéticos), y la energía consumida para la generación eléctrica y el propio sector energético, reportado en el Balance Nacional (Sener, 2007: 109-122). Para medir la pureza relativa del uso de la energía se utilizan dos indicadores: las emisiones totales y las emisiones per cápita. Como se observa en la figura 2, entre 1997 y 2006, las emisiones se incrementaron de 314 millones a 368 millones de toneladas de $\mathrm{CO}_{2}$, es decir que disminuyó la pureza del uso de la energía. Las emisiones per cápita ascendieron de 3.36 a 3.54 toneladas de $\mathrm{CO}_{2}$ por habitante entre 1997 y 2006, aunque son ligeramente inferiores a las de 1990, cuando alcanzaron 3.59 toneladas de $\mathrm{CO}_{2}$ por habitante. 
Figura 2.

Emisiones de $\mathrm{CO}_{2}$, asociadas al consumo de energía*

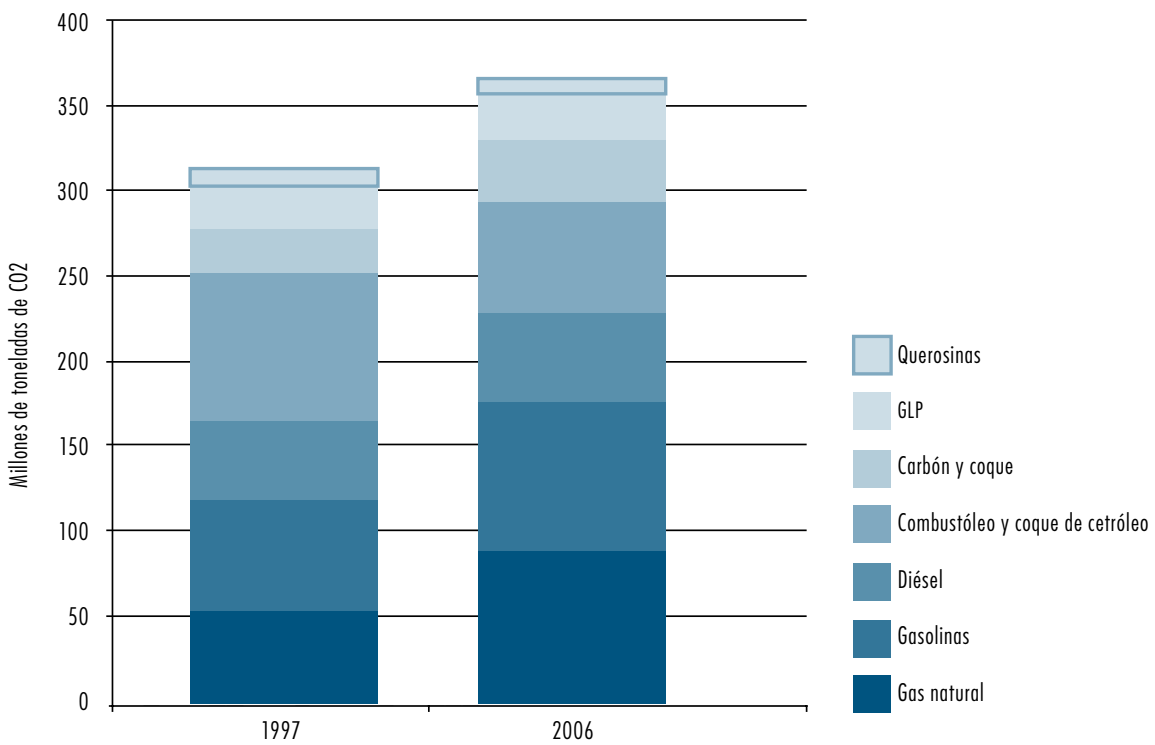

Fuente: elaboración propia con base en Sener (2007) e IPCC (1996).

*Incluye consumo de energía de los sectores de uso final, generación eléctrica y consumo propio del sector energético. No incluye emisiones fugitivas.

La participación de las energías renovables se define como el porcentaje de renovables respecto al consumo nacional total. La tabla 6 muestra cómo la participación de energías renovables, que incluyen hidroenergía, eoloenergía, geotermia, leña y bagazo de caña, pasaron de $12.5 \%$ a 11.3 por ciento.

tabla 6.

Participación de las fuentes renovables

de energía en el consumo (PJ)

\begin{tabular}{|l|r|r|}
\hline \multicolumn{2}{|c|}{1997} & 2006 \\
\hline Hidroenergía & 271.15 & 303.55 \\
Geoenergía & 56.08 & 66.96 \\
Eoloenergía & 0.04 & 0.45 \\
Leña & 247.00 & 247.20 \\
Bagazo & 91.37 & 94.24 \\
Otras fuentes & 4678.93 & 5600.16 \\
Total & 5344.57 & 6312.56 \\
Porcentaje de renovables & $12.5 \%$ & $11.3 \%$ \\
\hline
\end{tabular}

Fuente: elaboración propia con base en SENER (2007).

Incluye consumo de energía de los sectores de uso final, generación eléctrica y consumo propio del sector energético. 
El alcance recursos fósiles se estima como el cociente de las reservas de hidrocarburos (incluyendo probadas, probables y posibles) entre su producción bruta anual. De acuerdo con información de Pemex (Pemex, 1999 y 2007), en 1997 el alcance de hidrocarburos era de 40.5 años; para 2006 se redujo a 28.7 años. Este descenso se debió tanto al incremento de la producción de petróleo para exportación como a la ausencia de descubrimientos significativos, provocada por la caída en la inversión para exploración de crudo (tabla 7).

tabla 7.

Alcance de hidrocarburos

\begin{tabular}{|c|c|c|c|c|c|c|c|c|c|c|}
\hline & 1997 & 1998 & 1999 & 2000 & 2001 & 2002 & 2003 & 2004 & 2005 & 2006 \\
\hline Producción & 1504 & 1548 & 1433.8 & 1468.7 & 1493.6 & 1507.5 & 1587 & 1610.8 & 1604.2 & 1618.2 \\
\hline Reservas & 60900 & 60160 & 57741.2 & 58204.1 & 56154 & 52951 & 50032.2 & 48041 & 46914.1 & 46417.5 \\
\hline $\begin{array}{l}\text { Reservas/ } \\
\text { producción años }\end{array}$ & 40.5 & 38.9 & 40.3 & 39.6 & 37.6 & 35.1 & 31.5 & 29.8 & 29.2 & 28.7 \\
\hline
\end{tabular}

Producción y reservas en millones de barriles de petróleo crudo equivalente.

Fuente: Anuario Estadístico de Pemex (1999 y 2007).

\section{Normalización de los indicadores de sustentabilidad}

Para establecer una comparación de los indicadores, se propone una normalización básica entre los valores cero y uno. Es necesario mencionar que aun cuando algunos criterios de normalización pudieran parecer arbitrarios, lo sustantivo es la comparación entre los años de 1997 y 2006 respecto a determinados indicadores de sustentabilidad. El resultado no es importante por su valor absoluto, sino por la diferencia entre su valor de 1997 y el de 2006 y el criterio de sustentabilidad establecido. Es decir, interesa conocer la comparación entre ambos años, en relación con cuánto se alejan o se acercan al valor de sustentabilidad de cada indicador. Dado que la comparación se hace con el mismo criterio para ambos años, el resultado es totalmente válido. En el estudio de la CEPAL, por ejemplo, se busca la comparación entre países respecto a determinados criterios de sustentabilidad. En este caso, el estudio comparativo entre dos años clave del sector energético nacional busca explicar las consecuencias de la política energética en relación con ciertos indicadores de sustentabilidad. a continuaciónse presentan los criterios de normalización.

En el caso de la autarquía energética, el máximo valor de uno equivaldría a cero importaciones. De esta forma definimos el indicador de autarquía como la producción nacional, que equivale a la resta del valor máximo menos las importaciones. Desde nuestra perspectiva, es deseable y factible que el país produzca el total de la energía que consume. Así lo hizo México durante muchos años. 
tabla 8.

Comparación entre criterios de sustentabilidad energética

del sector energético nacional entre 1997 y 2006

\begin{tabular}{|l|l|l|}
\hline \multicolumn{2}{|c|}{$\mid 1997$} & 2006 \\
\hline Autarquía & 0.91 & 0.83 \\
Robustez & 0.91 & 0.62 \\
Exportaciones/PIB (\%) & 0.93 & 0.82 \\
Ingreso petrolero/total (\%) & 0.94 & 0.91 \\
Inversión con recursos propios & 0.86 & 0.13 \\
Productividad & 0.49 & 0.50 \\
Cobertura eléctrica & 0.93 & 0.97 \\
Satisfacción de necesidades básicas & 0.23 & 0.37 \\
Energía útil & 0.36 & 0.39 \\
Equidad (1-Gini)* & 0.34 & 0.35 \\
Pureza relativa & 0.95 & 0.86 \\
Participación de renovables & 0.06 & 0.06 \\
Alcance de hidrocarburos & 0.90 & 0.64 \\
Total ponderado & 0.67 & 0.61 \\
\hline
\end{tabular}

*El valor de 1997 es en realidad el de 2000.

La robustez se compone de tres indicadores: valor de las exportaciones de crudo respecto al PIB, participación del ingreso petrolero en el total, e inversión directa y a través de deuda en el sector petrolero. Para la normalización se asigna el valor arbitrario de $5 \%$ como porcentaje de las exportaciones respecto al PIB, y de 30\% como participación de los ingresos petroleros en los ingresos totales. Nuevamente, insistimos en la validez de estos valores, ya que si se consideran valores razonables lo relevante es la diferencia comparativa entre ambos años.

En el caso de la inversión a través de deuda se asigna el valor de uno a inversión sin deuda y cero a inversión total con deuda. Aun cuando se asignara un valor diferente para normalizar la deuda, nuevamente la diferencia entre ambos años sería la misma.

Para normalizar la productividad energética (inversa a la intensidad energética) se establece, de manera arbitraria, que el doble de lo que el país tiene actualmente equivaldría al valor máximo de uno (es decir, el objetivo es duplicar la productividad energética).

La normalización de la cobertura eléctrica se establece directamente. El total de hogares electrificados equivale al valor de uno.

La cobertura de necesidades energéticas básicas incluye dos indicadores, energía útil per cápita en el sector residencial, y equidad en el gasto de energía por deciles, 
para electricidad, gas y otros combustibles. En el primer caso, aun cuando la segunda Ley de la Termodinámica impide que toda la energía final se convierta en energía útil, pues no puede haber máquinas con el 100\% de eficiencia, lo cierto es que mientras más se acercan a este valor, mayor es la satisfacción de las necesidades. ${ }^{8}$ Esto es así por la construcción de este indicador. De esta forma la normalización equivaldría a uno, cuando la energía final es igual a la energía útil. Para normalizar la equidad en el consumo, se utiliza el valor de 1-Gini. Para estimar un solo valor del índice de Gini para todo el gasto de energía, se calcula un promedio ponderado por el porcentaje en el consumo residencial de cada combustible, incluida la leña. ${ }^{9}$ Para otorgar un solo valor, se le otorga un peso de $1 / 2$ a los dos indicadores.

La pureza relativa en el uso de la energía se normaliza haciendo la equivalencia del $95 \%$ de las emisiones de 1990 como la unidad. Esto se basa en el Protocolo de Kyoto. Pese a que México no se haya comprometido a disminuir emisiones de gases de efecto invernadero, sería deseable que pudiera cumplir con una estabilización de las emisiones a un valor 5\% menor que el de 1990, como lo establece el protocolo para diversos países denominados (Anexo 1), (Naciones Unidas, 1998: 1-25). De acuerdo con el Instituto Nacional de Ecología (1999: 3), para 1990 las emisiones de bióxido de carbono asociadas al uso de la energía fueron de 291 millones de toneladas de $\mathrm{CO}_{2}$. Para evaluar el indicador se utilizan las emisiones totales y las emisiones per cápita, cada una con un valor de 0.5. De esta forma, para 1997, las emisiones totales se alejaron $12 \%$ del valor que deberían haber tenido (por lo que su normalización es 0.88 ) y fueron $2 \%$ superiores en términos de las emisiones per cápita. Para 2006, las emisiones totales se alejaron 25\% (.75) y las emisiones per cápita 4\%. Así, respecto al máximo valor de uno, el de 1996 fue de 0.95, y el de 2006 se aleja, al alcanzar 0.86.

La participación de las energías renovables se normaliza bajo la premisa de que el máximo valor se obtendría si los renovables representaran al menos 50\% del consumo nacional de energía. Éste es el valor del indicador recomendado por la CEPAL.

El alcance de hidrocarburos se normaliza asumiendo el valor máximo de 45 años como el alcance de las reservas. Nuevamente, la importancia radica en el valor comparativo de ambos años, y es evidente que un mayor alcance implica mayores reservas y por tanto mejores condiciones del sistema energético nacional y menor vulnerabilidad del país.

8 La construcción de este indicador sólo señala la eficiencia del equipo y el combustible, pero no tiene en cuenta que la energía final puede destinarse a usos energéticos que no necesariamente implican la satisfacción de necesidades básicas.

9 Se incluye el consumo de leña aun cuando este combustible no necesariamente tenga un costo monetario en los hogares rurales, ya que puede ser obtenido por recolección. 
Los resultados para todos los indicadores se presentan en la tabla 8. El promedio de los indicadores resulta de 0.7 para 1997 y de 0.61 para 2006. La figura 3 muestra los valores normalizados de sustentabilidad energética para los años 1997 y 2006.

Figura 3.

Comparación entre criterios de sustentabilidad energética del sector energético nacional entre 1997 y 2006*

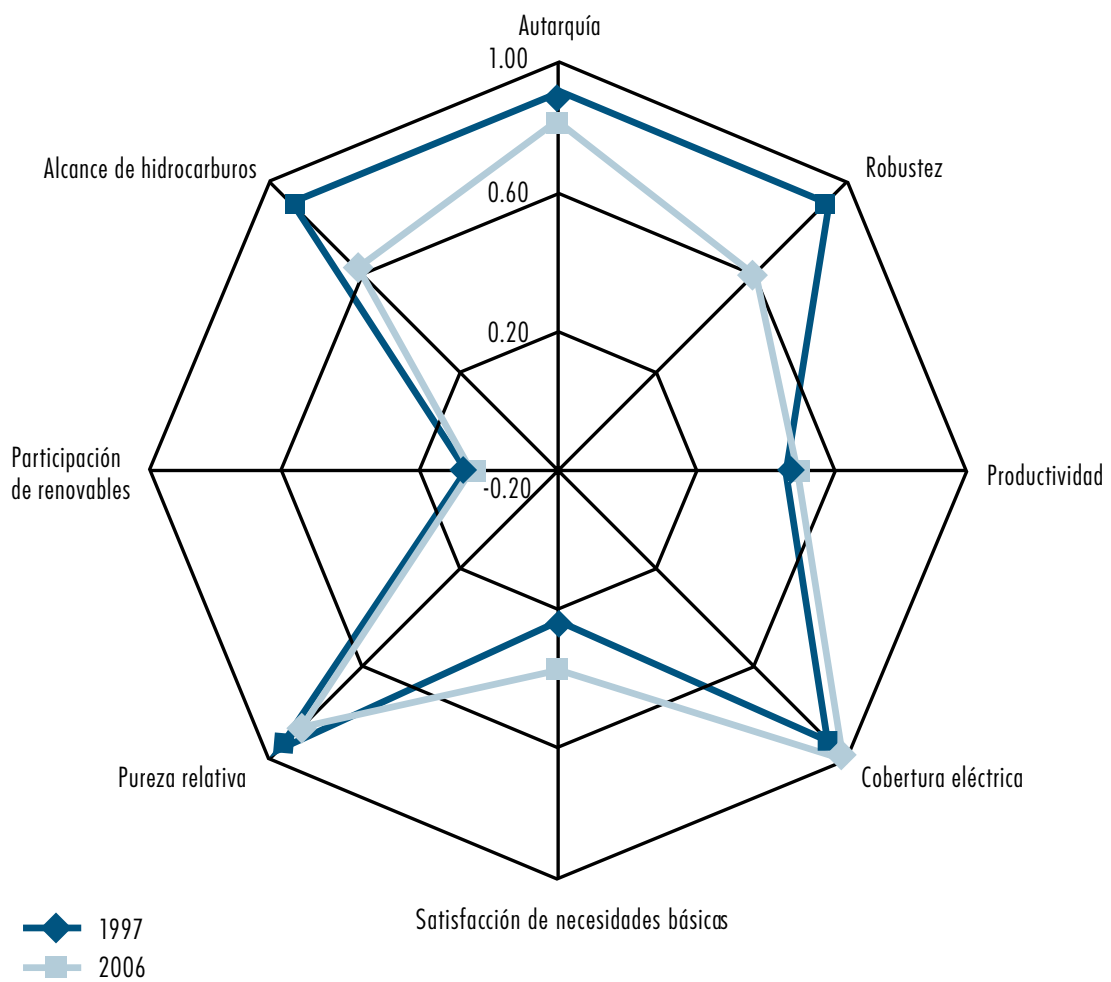

* A mayor área cubierta mayor acercamiento a los criterios de sustentabilidad energética.

\section{Discusión y conclusiones}

En las últimas décadas, la política energética nacional se ha concentrado en criterios de apertura del mercado y debilitamiento de los organismos públicos, en la sobreexplotación del recurso petrolero para exportación sin las inversiones necesarias para ampliar las reservas, en una visión de seguridad energética de América del Norte, la transición hacia el gas natural importado, y la utilización de Pemex y la CFE como instrumentos al servicio de las políticas de estabilidad macroeconómica. En este marco, se han debilitado los criterios de autarquía, robustez, transición energética hacia fuentes renovables, medio ambiente y satisfacción de necesidades básicas, criterios propuestos por las propias agencias internacionales de energía. 
Bajo la metodología planteada, que es una adaptación de la presentada por CEPAL et al. (2003), el sistema energético mexicano resulta ser menos sustentable en el año 2006 de lo que fuera en 1997. Aun cuando los indicadores de productividad, cobertura eléctrica y satisfacción de las necesidades energéticas básicas tienen valores mayores para 2006 que para 1997, y la participación de fuentes renovables permanece constante, los indicadores de autarquía, robustez, pureza relativa y alcance de hidrocarburos muestran una importante reducción, que hace que la sustentabilidad energética del sistema tenga una caída significativa. Desde esta perspectiva, considerando al valor de uno como el de mayor sustentabilidad, el sector energético mexicano pasó de 0.67 a 0.61 entre 1997 y 2006.

Mientras la política energética nacional no promueva mayor seguridad energética, menor participación del sector petrolero en las finanzas públicas, mayor inversión con recursos propios o menor apalancamiento en la realización de los proyectos o discriminación del endeudamiento del sector, así como una creciente participación de las fuentes renovables que se traduzca en el descenso de emisiones de $\mathrm{CO}_{2}$ y un incremento a la inversión en exploración y se reduzca la producción de hidrocarburos, la sustentabilidad del sector será cada vez menor.

\section{Referencias}

Calderón, Felipe, Primer informe de gobierno: Anexo estadístico, economía competitiva y generadora de empleo, vivienda, México, Presidencia de la República, 2 de septiembre de 2007.

Comisión para el Desarrollo Sustentable de las Naciones Unidas, Agenda 21, Nueva York, Naciones Unidas, 1992.

Comisión Económica para América Latina y el Caribe (CEPAL), Organización Latinoamericana de Energía (OLADE) y Sociedad Alemana de Cooperación Técnica (GTZ), Energía y desarrollo sustentable en América Latina: Enfoques para la política energética, Quito, CEPAL, 1997.

, Energía y desarrollo sustentable en América Latina: Guía para la formulación de políticas energéticas, Santiago de Chile, CEPAL, 2003, pp. 13-58.

Goldemberg, José et al., Energy for Development, Washington DC, World Resources Institute, $1987 \mathrm{a}$.
Energy for a Sustainable World, Washington DC, World Resources Institute, 1987b.

Instituto Nacional de Estadística y Geografía (INEGI). Sistema de Información Económica [http://dgcnesyp.inegi.org.mx/bdiesi/ bdie.html].

Instituto Nacional de Estadística y Geografía. Encuesta Nacional de Ingreso Gasto en los Hogares 2000 y 2004 [http://www.inegi. gob.mx].

International Atomic Energy Agency (IAEA), United Nations Department of Economic and Social Affairs (UNDESA), International Energy Agency (IEA), Eurostat y European Energy Agency (EEA), Energy Indicators for Sustainable Development: Guidelines and methodologies, Viena, IAEA, 2005.

Instituto Nacional de Ecología (INE), Inventario nacional de emisiones de gases de efecto invernadero con cifras de 1990, México, INE-Semarnat, 1999 [http://www.ine.gob. mx/cclimatico/descargas/inv1990.pdf].

Vol. 40, núm. 158, julio-septiembre / 2009 
Intergovernemntal Pannel of Climate Chage (IPCC), Revised 1996 IPCC Guidelines for National Greenhouse Gas Inventories: Reference Manual, vol. 3, Ginebra, World Meteorological Organization-United Nations Environment Programme, 1996,

OLADE, CEPAL, GTZ, Energía y desarrollo sustentable en América Latina y el Caribe: Guía para la formulación de políticas energéticas, Quito, OLADE, julio de 2000.

Organización de las Naciones Unidas, Protocolo de Kyoto de la Convención Marco de las Naciones Unidas sobre el Cambio Climático, Nueva York, ONU, 1998.

http://unfccc.int/resource/docs/convkp/kpspan. pdf.

Pemex, Anuario estadístico, México, 1999 a 2007 [http://www.pemex.com/index.cfm? action $=$ content $\&$ sectionID $=2 \&$ catid $=262$ $4 \&$ contentID=2633].
Salgado, René y Hugo Altomonte, Indicadores de sustentabilidad 1990-1999, Santiago de Chile, CEPAL, División de Recursos Naturales e Infraestructura, 2001. [http://www. eclac.cl/drni/proyectos/energ\%C3\%ADa/ indicadores/indicadores.pdf].

Secretaría de Energía (Sener), Balance nacional de energía 2006, México, Sener, 2007.

UNDESA, Indicators of Sustainable Development: Guidelines and Methodologies, 2a ed., Nueva York, septiembre de 2001.

UNDESA, World Commission on Environment and Development (WCED), Our Common Future, ONU, Oxford University Press, 1988. 
Anexo

Tabla A.

Indicadores energéticos para el desarrollo sustentable de acuerdo con la IEAA et al.

\begin{tabular}{|c|c|c|}
\hline Tema & Sub tema & Indicador \\
\hline Equidad & $\begin{array}{l}\text { Accesibilidad } \\
\text { Comodidad } \\
\text { Disparidad }\end{array}$ & $\begin{array}{l}\text { Porcentaje de la población sin electricidad o acceso a energía comercial } \\
\text { Porcentaje del ingreso destinado a combustibles y electricidad } \\
\text { Uso de energía residencial por cada nivel de ingreso y combustible }\end{array}$ \\
\hline Salud & Seguridad & Accidentes fatales en la cadena energética \\
\hline $\begin{array}{l}\text { Patrones de producción } \\
\text { y uso }\end{array}$ & $\begin{array}{l}\text { Uso global } \\
\text { Productividad global } \\
\text { Eficiencia en el suministro } \\
\text { Producción } \\
\text { Usos finales } \\
\text { Diversificación }\end{array}$ & $\begin{array}{l}\text { Energía per cápita } \\
\text { Energía por unidad de Producto Interno Bruto } \\
\text { Eficiencia en la conversión y distribución } \\
\text { Razón reservas/producción } \\
\text { Razón recursos/producción } \\
\text { Intensidad energética de los diversos sectores de uso final (industrial } \\
\text { transporte, residencial, agropecuario, etc.) } \\
\text { Porcentaje de las diversas fuentes primarias de energía en el consumo } \\
\text { Porcentaje de las fuentes que no son de origen fósil } \\
\text { Porcentaje de las fuentes renovables de energía } \\
\text { Precios }\end{array}$ \\
\hline Seguridad & $\begin{array}{l}\text { Importaciones } \\
\text { Reservas }\end{array}$ & $\begin{array}{l}\text { Energía neta importada } \\
\text { Reservas de las diferentes fuentes de energía vs. consumo }\end{array}$ \\
\hline Atmósfera & $\begin{array}{l}\text { Cambio climático } \\
\text { Calidad del aire }\end{array}$ & $\begin{array}{l}\text { Emisiones de } \mathrm{CO}_{2} \text { totales, per cápita y por PIB } \\
\text { Concentración de contaminantes en la atmósfera de zonas urbanas } \\
\text { Contaminantes atmosféricos debidos a los sistemas energéticos }\end{array}$ \\
\hline Agua & Calidad del agua & Descargas de los sistemas energéticos \\
\hline Suelo & $\begin{array}{l}\text { Calidad de suelo } \\
\text { Bosques } \\
\text { Generación y manejo de } \\
\text { residuos sólidos }\end{array}$ & $\begin{array}{l}\text { Área en donde la acidificación está por encima de la capacidad de carga } \\
\text { Deforestación asociada a uso de la energía } \\
\text { Tasa de generación de residuos sólidos a energía producida } \\
\text { Porcentaje de disposición adecuada de residuos sólidos respecto al } \\
\quad \text { total de residuos } \\
\text { Tasa de generación de residuos radioactivos respecto a energía generada }\end{array}$ \\
\hline
\end{tabular}

Fuente: (IEAA, UNDESA, IEA, Eurostat, EEA, 2005).

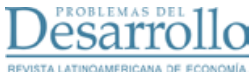


Tabla B.

Indicadores de sustentabilidad energética de acuerdo con CEPAL et al.

\begin{tabular}{|c|c|c|}
\hline Indicador & $\begin{array}{l}\text { Alta sustentabilidad } \\
\text { relacionada con: }\end{array}$ & Responde a objetivos \\
\hline Autarquía energética & $\begin{array}{l}\text { Baja participación de las } \\
\text { importaciones en la oferta } \\
\text { energética }\end{array}$ & $\begin{array}{l}\text { Seguridad del abastecimiento externo } \\
\text { Sostenimiento del espacio de maniobra para la políitca } \\
\text { Reducción del riesgo de desequilibrio en la balanza de pagos }\end{array}$ \\
\hline $\begin{array}{l}\text { Robustez frente a } \\
\text { cambios externos }\end{array}$ & $\begin{array}{l}\text { Baja contribución de las } \\
\text { exportaciones energéticas } \\
\text { al PIB }\end{array}$ & $\begin{array}{l}\text { Flujos estables de ingresos de las exportaciones } \\
\text { Menor peso de ingresos variables en el presupuesto } \\
\text { Reducción del riesgo de desequilibrio en la balanza de pagos }\end{array}$ \\
\hline Productividad energética & $\begin{array}{l}\text { Alto PIB por unidad } \\
\text { de energía }\end{array}$ & $\begin{array}{l}\text { Eficiencia productiva } \\
\text { Eficiencia energética } \\
\text { Financiamiento suficiente (por reducción de necesidades de inversión } \\
\text { del sector) } \\
\text { Reducción de costos del suministro energético } \\
\text { Abastecimiento suficiente (por reducción de la demanda) } \\
\text { Mejor calidad del aire (reducción de emisiones de contaminantes locales) } \\
\text { Reducción de emisiones de gases de efecto invernadero } \\
\text { Extensión de alcance de recursos no renovables }\end{array}$ \\
\hline Cobertura eléctrica & $\begin{array}{l}\text { Alto porcentaje de hogares } \\
\text { electrificados }\end{array}$ & $\begin{array}{l}\text { Diversificación del mix energético } \\
\text { Abastecimiento suficiente } \\
\text { Acceso a energéticos modernos y productivos } \\
\text { Abastecimiento de servicios sociales }\end{array}$ \\
\hline $\begin{array}{c}\text { Cobertura de necesidades } \\
\text { energéticas básicas }\end{array}$ & $\begin{array}{l}\text { Suficiente consumo de energía } \\
\text { útil residencial }\end{array}$ & $\begin{array}{l}\text { Satisfacción de necesidades básicas } \\
\text { Diversificacion del mix energético } \\
\text { Uso sustentable de la leña }\end{array}$ \\
\hline $\begin{array}{l}\text { Pureza relativa del uso } \\
\text { de la energía }\end{array}$ & $\begin{array}{l}\text { Bajos niveles de emisiones } \\
\text { de } \mathrm{CO}_{2}\end{array}$ & $\begin{array}{l}\text { Mejor calidad del aire (reducción de emisiones de contaminantes locales) } \\
\text { Reducción de emisiones de gases de efecto invernadero }\end{array}$ \\
\hline $\begin{array}{l}\text { Uso de energías } \\
\text { renovables }\end{array}$ & $\begin{array}{l}\text { Alta participación de energías } \\
\text { renovables en la oferta } \\
\text { energética }\end{array}$ & $\begin{array}{l}\text { Mejor calidad del aire (reducción de emisiones de contaminantes locales) } \\
\text { Reducción de emisiones de gases de efecto invernadero }\end{array}$ \\
\hline $\begin{array}{l}\text { Alcance recursos fósiles } \\
\text { y leña }\end{array}$ & & $\begin{array}{l}\text { Extensión de recursos a largo plazo } \\
\text { Seguridad de suministro a largo plazo } \\
\text { Mantenimiento de un mínimo de patrimonio natural }\end{array}$ \\
\hline
\end{tabular}

Fuente: CEPAL, OLADE, GTZ (1997). 